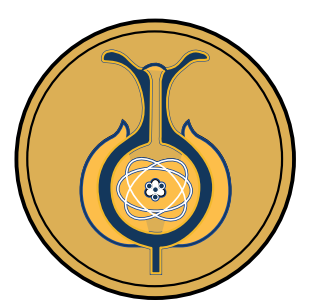

\title{
La eficacia pedagógica en la cultura organizativa escolar y la comunidad profesional de aprendizaje
}

\author{
Pedagogical effectiveness in the school organizational culture and the \\ professional learning community
}

A eficácia pedagógica na cultura organizacional escolar e a comunidade profissional de aprendizagem

\section{Derling José Mendoza Velazco ${ }^{1}$ Magda Francisca Cejas Martínez ${ }^{2,3} \cdot$ Mercedes Navarro Cejas $^{4} \cdot$ Elizeth Mayrene Flores Hinostroza ${ }^{1}$ Karina Maribel Castillo Pinos ${ }^{1}$}

Received: Apr/29/2020 • Accepted: Sep/11/2020 Published: Jul/31/2021

\section{Resumen}

El objetivo del presente estudio fue analizar la eficacia docente en la cultura organizativa de las escuelas y la comunidad profesional de aprendizaje. La investigación fue de carácter cuantitativo, con una muestra constituida por muestreo aleatorio simple de 359 profesores que trabajaban en escuelas de nivel inicial, básico y general unificado de las ciudades de Cuenca y Azogues, Ecuador. Se aplicó como instrumentos cuatro cuestionarios tipo Likert de cinco opciones de respuesta, con el método de análisis de prueba t, F y Sheffe. Mediante el análisis de correlación y regresión múltiple, los resultados indicaron que los maestros responden a la organización de la escuela, según sus características personales. La cultura organizativa de la escuela se está transformando gradualmente, en una cultura positiva e ideal. La comunidad profesional de aprendizaje tuvo un efecto positivo en la eficacia de los maestros. Como conclusión, la cultura organizativa de la escuela y la comunidad profesional de aprendizaje debe ir más allá de la dimensión interactiva y pasar a la dimensión de la integración como elementos de la mejora de la escuela para obtener una mayor eficacia pedagógica.

Palabras clave: escuela; cultura organizativa; comunidad de aprendizaje; eficacia pedagógica

\section{Abstract}

The objective of this study was to analyze teacher effectiveness in the organizational culture of schools and the professional learning community. This was a quantitative research, with a simple random sample of 359 elementary teachers at the initial, basic, and general unified level in the cities of Cuenca and Azogues, Ecuador. Four Likert-type questionnaires of five answer options were applied as instruments, with the t, F, and Scheffé test

Derling José Mendoza Velazco, \derling969@gmail.com,(ㄱ https://orcid.org/0000-0001-8275-3687

Magda Francisca Cejas Martínez, $\$ magdacejas09@gmail.com, (D) https://orcid.org/0000-0002-0618-3608

Mercedes Navarro Cejas, $\$ ur.mercedesnavarro@uniandes.edu.ec, (1) https://orcid.org/0000-0003-4377-7250

Elizeth Mayrene Flores Hinostroza, $₫$ elizethfloress2005@gmail.com, (D https://orcid.org/0000-0003-2171-8348

Karina Maribel Castillo Pinos, $\triangle$ karina.castillo@unae.edu.ec, (10 https://orcid.org/0000-0002-1154-1297

1 Educación en Ciencias Experimentales, Universidad Nacional de Educación, Chuquipata, Ecuador.

2 Facultad de Ciencias Políticas y Administrativas, Universidad Nacional de Chimborazo, Riobamba, Ecuador.

3 Departamento de Ciencias Económicas Administrativas y de Comercio, Universidad Nacional de las Fuerzas Armadas, Latacunga, Ecuador.

4 Departamento de Investigación, Universidad Regional Autónoma de los Andes, Riobamba, Ecuador. 
analysis method. Using correlation and multiple regression analysis, results indicated that teachers respond to school organization according to their personal characteristics. The school organizational culture is gradually being transformed into a positive and ideal culture. The professional learning community had a positive effect on teachers' effectiveness. In conclusion, the organizational culture of the school and the professional learning community should go beyond the interactive dimension and move towards the integration dimension to improve the school in order to obtain greater pedagogical effectiveness.

Keywords: school; organizational culture; learning community; pedagogical effectiveness

\section{Resumo}

0 presente estudo teve como objetivo analisar a eficácia docente na cultura organizacional das escolas e na comunidade profissional de aprendizagem. A pesquisa foi de caráter quantitativo, com um exemplar constituído de amostragens aleatórias simples de 359 professores que trabalhavam em escolas de nível inicial, básico e geral unificado das cidades de Cuenca e Azogues, no Equador. Foram aplicados, como instrumento, quatro questionários tipo Likert de cinco opções de resposta com o método de análise de teste $t, F$ e Sheffe. Por meio da análise de correlação e regressão múltipla, os resultados indicaram que os professores respondem à organização da escola, segundo suas características pessoais. A cultura organizacional da escola está se transformando gradativamente em uma cultura positiva e ideal. A comunidade profissional de aprendizagem teve um efeito positivo na eficácia dos professores. Em conclusão, a cultura organizacional da escola e a comunidade profissional de aprendizagem deve ir além da dimensão interativa e passar à dimensão da integração como elementos de melhoria da escola para obter uma maior eficácia pedagógica.

Palavras-chave: escola; cultura organizacional; comunidade de aprendizagem; eficácia pedagógica

\section{INTRODUCCIÓN}

Dentro de las organizaciones escolares, existe un alto nivel de interés en la aplicación de las funciones, ya que los profesores tienen un papel clave de responsabilidad en la educación. Por ejemplo, un profesor con una alta autoeficacia tiene grandes expectativas en cuanto a la mejora y el progreso de los estudiantes, asume la responsabilidad del aprendizaje de sus alumnos, establece objetivos y estrategias de aprendizaje y trata de encontrar métodos de enseñanza eficaces.

Además, los maestros con alta eficacia exhiben altos niveles de aptitudes y conocimientos especializados y alcanzan los objetivos de la educación, incluidos los logros de los estudiantes, a pesar de los entornos y las situaciones difíciles. Así como la autoeficacia afecta al rendimiento de las personas, la eficacia de un profesor afecta a su rendimiento en la enseñanza y a las actividades diarias en general (Aditomo y Klieme, 2020).

Es necesario que haya más estudios e investigaciones sobre cómo aumentar la autoeficacia de los maestros en las provincias de El Cañar y Azuay, teniendo en cuenta la participación en actividades de desarrollo profesional, como una variable importante que la afecta (Aydeniz y Dogan, 2016). Esta se desarrolla a través de una autoevaluación positiva mediante experiencias exitosas. 
Por lo tanto, la eficacia del maestro puede mejorarse participando en actividades de desarrollo profesional, en las cuales no solo adquieren los conocimientos y las aptitudes que subyacen a una experiencia exitosa, sino que también crean sinergia en la solución de problemas, mediante la interacción con colegas, y reciben, elogian y alientan mediante la participación en el proceso.

A este respecto, el presente estudio comienza por superar las limitaciones de la teoría del liderazgo de la autoeficacia (plantadas en estudios anteriores, sobre las comunidades de aprendizaje profesional) que está estrechamente relacionada con la eficacia de los maestros, y se centra en el hecho de que el liderazgo ha sido recientemente, objeto de debate y discusión. La publicación de Ross y Bruce (2007), determinó que la participación en los programas de desarrollo profesional mejoraba la autoeficacia, especialmente, la en la gestión del aprendizaje.

Sin embargo, es difícil que las comunidades de aprendizaje profesional se establezcan adecuadamente, o se formen plenamente, en comparación con el grado en que se hace hincapié en ellas, dentro del ámbito escolar. En un estudio realizado por Benedict y Aram (2017), se comprobó que, si bien los maestros de los Estados Unidos aceptan el concepto de una comunidad profesional de aprendizaje, tienen poca capacidad para poner en práctica y aplicar realmente el concepto en su entorno.

La razón es que no basta con cambiar la estructura administrativa de la escuela como forma de mejorarla, sino que es necesario cambiar la conciencia de los integrantes, lo cual no es fácil (Areepattamannil, 2012). En otras palabras, para Fogleman, McNeill y Krajcik (2011) cambiar la estructura sin cambiar el sistema de creencias no puede hacer un cambio fundamental.
Por lo tanto, este estudio trata de encontrar formas de ampliar la eficacia del profesorado examinando cómo varía esta, según la cultura de la organización escolar (COE) y el grado de la comunidad profesional de aprendizaje (CPA) en las ciudades de Azogues y Cuenca de la República del Ecuador, durante el ciclo académico 2019-2020.

\section{MARCO TEÓRICO}

\section{La cultura organizativa escolar}

Las organizaciones tienen diferentes culturas, cada una con un propósito y actividades diferentes. La COE se refiere a los supuestos, las creencias, los valores, las normas y las costumbres, los hábitos y los rituales que los integrantes de la organización comparten en el proceso de adaptación al entorno externo y de resolución de problemas internos.

La COE se refiere a la que está dentro de la institución (Wieslander, 2019 y Lee, 2007), en otras palabras, significa que es única de la escuela. El interés de la academia en esta cultura, se debe a los continuos informes de que esta tiene un efecto directo $\mathrm{o}$ indirecto en la efectividad organizacional de la escuela. El concepto de COE es útil para explicar el comportamiento de los integrantes de la organización escolar desde una perspectiva más amplia y a largo plazo (Zanin y Bisel, 2020).

Una forma de entender la COE es acercarse a través de la categorización, sin embargo, esto no es fácil, ya que implica una combinación de varios factores en juego, $\mathrm{y}$ el hecho de que se haga, también es controvertido. En este estudio, el criterio de división conceptual se basó en lo establecido por Huopalainen (2019), al indicar que la adaptación al entorno externo de la institución, es el 
proceso para abordar los problemas internos. Por lo tanto, la dimensión conductual al ambiente externo se divide activa y pasivamente, y el proceso de resolver los problemas internos en la flexibilidad y la rigidez.

Los tipos de cultura se dividieron en racional, de grupo, innovadora y de jerarquía, como se presenta en figura 1 (Lee, 1997). La cultura de la innovación busca aplicar nuevos métodos de enseñanza desarrollando ideas y programas educativos de manera entusiasta y exploratoria en respuesta a los cambios del entorno externo. La cultura racional responde activamente, a los cambios en el ambiente externo, enfatiza el logro de las metas educativas en las operaciones escolares y considera las consecuencias del éxito o el fracaso, pero muestra rigidez en la resolución de problemas.

La cultura de grupo es pasiva en los cambios del entorno externo, pero hace hincapié en el trabajo en equipo o la cooperación mutua y muestra flexibilidad en el proceso de solución de problemas. La cultura jerárquica es pasiva en el entorno externo y el funcionamiento de la escuela se gestiona bajo la dirección del director o el vicedirector, según los procedimientos y los reglamentos.

\section{Comunidad profesional de aprendizaje}

En cuanto a la innovación en las escuelas, en los Estados Unidos y otros países europeos, se han venido celebrando debates sobre la comunidad profesional de aprendizaje (CPA) de los maestros y la aplicabilidad del sitio de la escuela, durante los últimos 20 años. En particular, en ese país, como parte de los esfuerzos por innovar los sitios escolares, la aplicabilidad de estas comunidades, se ha llevado a cabo rápidamente, entre los educadores y los administradores (Vinhote et al., 2020 y Fogleman et al., 2011).

La ciudad de Quito intentó muchos enfoques conceptuales en la dirección de la innovación escolar y reconoció la necesidad de formar una CPA que pueda lograr el crecimiento del desarrollo de los maestros en conjunto como grupo y no en el nivel individual, según Mendoza, et al., (2019a).

Una CPA es una organización que mejora continuamente la capacidad de resolución de problemas de la organización, mediante el intercambio de nuevos conocimientos adquiridos por los individuos en el nivel de la organización (Rodríguez, et al., 2009, DuFour, et al., 2010 y Senge, et al., 2000).

En conclusión, el concepto de CPA en este estudio, significa una comunidad en la que los maestros exploran y mejoran continuamente, todas las áreas de las actividades de enseñanza-aprendizaje mediante el aprendizaje activo y la cooperación con sus colegas, con el fin de lograr el crecimiento de los estudiantes y la promoción del aprendizaje (Elkader, 2014 y Robin, 2004).

\begin{tabular}{c|c|c|c}
\multirow{3}{*}{ Pasivo } & III & I \\
& Cultura de grupo & Cultura de la innovación & \multirow{2}{*}{ Activo } \\
\cline { 2 - 3 } & IV & II \\
\hline \multirow{2}{*}{ Cultura de jerarquía } & Cultura racional & \\
\hline \multicolumn{2}{|c|}{ Rigidez }
\end{tabular}

Figura 1. Clasificación de los tipos de cultura organizativa de la escuela. 


\section{Objetivo de la investigación}

El objetivo del presente estudio fue analizar la eficacia pedagógica de la cultura organizativa de las escuelas y de la comunidad profesional de aprendizaje en las ciudades de Azogues provincia del Cañar y Cuenca Provincia de Azuay de la República del Ecuador.

\section{METODOLOGÍA}

La investigación se fundamenta en el paradigma cuantitativo, establecido y aceptado por un número sustancial de personas en una comunidad de estudio. Este tipo de investigación, se utiliza tanto en ciencias naturales como sociales, dependiendo de su área de estudio y tema específico, se deberá analizar las metodologías que generalmente, se utilizan para llevarla a cabo, según Kivunja y Bawa (2017) y Mendoza, et al., (2019b). También implica, cuestionarios, pruebas y análisis de contenido estructurado, y los resultados se obtienen mediante técnicas estadísticas, matemáticas o computarizadas por softwares operativos.

\section{Población y muestra}

Para llevar a cabo este estudio, se encuestó a 370 docentes de un total de 15 escuelas ubicadas en la ciudad de Azogues provincia del Cañar y Cuenca provincia de Azuay, República del Ecuador, durante el ciclo académico de agosto 2019-marzo 2020. La población fue constituida por 500 educadores, quienes laboran en esas ciudades. Además se utilizó un método de muestreo aleatorio considerando la localidad de la escuela.

Los cuestionarios fueron enviados mediante correos institucionales a las escuelas objetos de estudio. De los 370 cuestionarios que se recogieron, aproximadamente, el $89,7 \%$ fue considerado para el estudio, descartando los cuestionarios que no se completaron, es decir se utilizaron los datos de 359 docentes para el análisis de los resultados considerándose, la selección de la muestra de estudio.

\section{Instrumentos}

\section{Instrumento de medición para la cultura organizativa de las escuelas}

En este estudio, se aplicó un análisis cuantitativo para la medición de la COE de forma empírica y práctica. Por lo tanto, el cuestionario de COE utilizó un cuestionario diseñado de acuerdo a la situación de las escuelas objetos de estudio. La estructura del cuestionario para analizar la $\mathrm{COE}$, se divide en tres partes; la primera es la cultura de innovación con los siguientes ítems:

- ¿Cuánto consideras que tu institución es innovadora?

- ¿Qué nivel de acceso tienes en tu trabajo, en cuanto a recursos tecnológicos, equipos de labor, herramientas de investigación, materiales ineludibles para realizar tu labor apropiadamente?

- ¿Cuánto valora tu institución, los alcances y las publicaciones aplicadas en tus estudios?

- ¿Cuánta inspiración emite tu trabajo para seguir laborando, al percibir su misión y visión organizativa?

- ¿Cuánto favorece tu institución educativa a los objetivos de la innovación escolar?

Los ítems de la segunda sección para el estudio de la cultura racional fueron:

- ¿Cuánto permiten tus superiores mejorar tus habilidades profesionales? 
- ¿Qué áreas de entrenamiento existen en tu en tu institución para descansar en los momentos libres?

- ¿Qué tan respetado te sientes por tu equipo y tu organización?

- ¿Cuánta cultura organizacional emite tu ambiente laboral?

- ¿Cuánto celebra la organización escolar tus logros y metas de profesionalización?

En la tercera sección, para el estudio de la cultura de grupo, se aplicaron los siguientes ítems:

- ¿Qué libertad te brindan tus superiores institucionales para solventar cómo efectuar tu trabajo?

- ¿Cuánto se preocupan tus compañeros por darte retroalimentación?

- ¿Qué cómodo te sientes físicamente en tu lugar de trabajo?

- ¿Cuánto te alienta la organización escolar de tu institución, para discutir y contribuir ideas?

- ¿Cuántos docentes lideres existen en tu institución?

En la cuarta sección cultura jerárquica, se encuentran los siguientes ítems:

- ¿Qué tal es tu relación con tu jefe inmediato?

- ¿Cuánto apoyo o ayuda inmediata recibes de tus compañeros cuando tienes inconvenientes laborales?

- ¿Qué tan confiable, amable y sincero es tu jefe inmediato?

- ¿Cuántos amigos tienes en el trabajo?

- ¿Cuánta orientación recibes de tu director, rector, coordinador o jefe, en momentos que presentan dificultades?
Las opciones de respuesta para cada ítem del cuestionario fueron de tipo escala, con 5 puntos, en la opción Siempre (S), con la valoración más alta (5), luego la opción Casi siempre (CS) con el puntaje (4), opción intermedia neutral Algunas veces (AV), con valor de (3), opción Casi nunca (CN), con puntaje de (2), finalmente, la opción de menor valor, Nunca $(\mathrm{N})$ con un puntaje de (1).

Para el instrumento se confirmó la fiabilidad y la validez del cuestionario final. Los valores de alfa de Cronbach de la herramienta de medición utilizada en este estudio mostraron una cultura de innovación de 0,810 , una racional de 0,780 , una de grupo de 0,850 y una de jerarquía de 0,850 .

\section{Instrumento de medición para la comunidad profesional de aprendizaje}

En el segundo instrumento para analizar la CPA, se diseñó un cuestionario en el que se desarrollaron 16 ítems. El cual se estructura en cuatro secciones.

En la primera sección para analizar la promoción del aprendizaje, se aplicaron los siguientes ítems:

- ¿Qué aprendizaje se brinda de forma colectiva en su institución?

- ¿Cuánto apoyo emite en valores y visiones la institución educativa en el aprendizaje estudiantil?

- ¿Cuántos cambios institucionales existen para facilitar el desarrollo del aprendizaje?

- ¿Qué trato brinda el profesorado al alumnado?

En la segunda sección para el estudio de la cultura cooperativa, se desarrollaron los siguientes ítems: 
- ¿Qué condiciones de apoyo reciben de la institución?

- ¿Qué interrelaciones epistémicas y sociales existen con otras escuelas?

- ¿Cuáles decisiones son analizadas exterior o externamente en su institución educativa, por lo que está sucediendo de manera interna?

- ¿Qué nivel de consideración toma el personal académico directivo con los integrantes de la comunidad profesional de aprendizaje?

En la tercera sección para describir la investigación en grupo, se aplicaron los siguientes ítems:

- ¿Cuántas investigaciones grupales Permite la institución?

- ¿Cuánto apoyo económico brinda la institución en las investigaciones?

- ¿Qué cantidad de horas están destinadas en el cronograma para la investigación?

- ¿Cuántos talleres o cursos otorga la institución para la actualización investigativa?

En la cuarta sección para el análisis descriptivo de la práctica pedagógica, se aplicaron los ítems:

- ¿Qué frecuencia publicitaria aplica la institución de las practicas pedagógicas, para para contribuir a la formación académica individual y organizacional?

- ¿Cuál es el nivel de aprendizaje de los estudiantes al aprender directamente de las practicas pedagógicas?

- ¿Cuánta importancia le tiene a la educación?
- $\quad$ ¿Cuál es el rango de aporte que usted otorga a los estudiantes para brindar sus propias técnicas o métodos de resolución de problemas durante las practicas pedagógicas?

En el cuestionario dos, se aplicó una escala de cinco puntos tipo Likert, con las mismas opciones de respuesta que el cuestionario uno, en el estudio se desarrolló la herramienta de medición con el coeficiente alfa de Cronbach, los cuales fueron todos superiores a 0,800 , lo que confirma su fiabilidad. El alfa Cronbach de la herramienta de medición utilizada en este estudio se demostró que la promoción del aprendizaje fue de 0.850 , la cultura de cooperación de 0.900, los grupos de investigación de 0.930 y práctica pedagógica de 0.960 .

\section{Instrumentos de medición de la efi- cacia pedagógica de los profesores}

Para medir la eficacia pedagógica, se diseñó un cuestionario estructurado en 3 secciones, cada una con 4 ítems, con un total de 12. Los ítems de la primera sección fueron destinados para el análisis de la confianza, los cuales fueron los siguientes:

- ¿Cuánto confía en los demás?

- ¿Qué nivel de confianza tiene en los demás?

- ¿Cuál es el rango de moralidad que tienen sus compañeros de trabajo?

- ¿Cuál es el nivel de funcionamiento efectivo de su institución?

Los ítems de la sección autorregulación, fueron los siguientes:

- ¿Cuántos objetivos y control pones a tu progreso? 
- ¿Cuántos planes haces para poder alcanzar los objetivos que te gusta?

- ¿Cuánto aprendes de tus errores?

- ¿Qué nivel de voluntad posees?

Los ítems de la sección dificultad de la tarea fueron los siguientes:

- ¿Qué tan acorde te sientes con tu tiempo para el desempeño educativo?

- ¿Cuántas actividades te brindan en tu institución?

- ¿Cuánto podrías mejorar tu actitud educativa?

- ¿Cuál eficiente es la evaluación que realiza a sus alumnos para generar motivación?

A estas preguntas se les aplicó una escala Likert de 5 puntos, y cuanto más alta era la puntuación, más alto era el nivel de eficacia del profesor, y las puntuaciones medidas se sumaron y luego se evaluaron. El coeficiente estadístico alfa de Cronbach para la herramienta de medición utilizada en este estudio fue de 0,780 para la confianza, 0,870 para la autorregulación, 0,780 para el desafío.

Tabla 1. Cuestionario de eficacia pedagógica de los profesores.

\begin{tabular}{llccc}
\hline & Categoría & $\mathbf{N}$ & $\mathbf{6}$ & $\mathbf{\sigma}$ \\
\hline Organización cultural escolar & Cultura organizacional & 359 & 3.81 & .69 \\
& Cultura de la innovación & 358 & 3.68 & .70 \\
& Cultura racional & 357 & 2.76 & .81 \\
& Cultura de la jerarquía & 357 & 2.58 & .86 \\
Comunidad profesional de aprendizaje & Promoción del aprendizaje & 358 & 4.05 & .59 \\
& Cultura cooperativa & 358 & 4.09 & .64 \\
& Grupo de investigación & 355 & 3.77 & .75 \\
& Práctica pedagógica & 356 & 3.86 & .70 \\
& Total & 351 & 3.91 & .64 \\
& Confianza & 350 & 3.52 & .61 \\
& Autorregulación & 349 & 3.46 & .60 \\
& Dificultad de la tarea (desafío) & 349 & 3.70 & .67 \\
& Total & 348 & 3.54 & .55 \\
\hline
\end{tabular}

\section{Procedimiento de análisis de datos}

El método estadístico de análisis de los datos reunidos mediante el cuestionario fue el siguiente:

En primer lugar, para examinar las diferencias entre las variables de la investigación, según las características individuales de los profesores, se utilizó la prueba $\mathrm{F}$ y Scheffe, métodos recomendados por Zaigraev y Wilk, (2020). Luego para averiguar si hay una diferencia en las escuelas, según el grado de actividad en las CPA, comparando las escuelas con y sin CPA activas, se utilizó el método de la prueba t. Finalmente, se utilizó el análisis de correlación y regresión múltiple, para analizar qué variables de la COE y de la CPA tenían un efecto significativo en la eficacia de los maestros.

\section{ANÁLISIS Y RESULTADOS \\ Tendencias generales en la cultu- ra organizativa de las escuelas, la comunidad profesional de aprendi- zaje y la eficacia de los maestros}

Los resultados de cómo los maestros generalmente, perciben la COE, la CPA y la eficacia, se presentan en la tabla 1. 
Para conocer el grado de reconocimiento y percepción de los profesores, los resultados se convirtieron en una escala de 5 puntos y luego se compararon. En el caso de la COE, la de grupo fue la más alta con 3,81 , la de innovación 3,68, la racional 2,76 y la de jerarquía 2,58. Este resultado es contrario a los de estudios anteriores, como los plateados por Lee (2007), quien reconoció que la cultura jerárquica es alta entre los tipos de COE.

La razón por la que los resultados en el presente estudio son diferentes del pasado, es que la cultura escolar está cambiando gradualmente, al tiempo que se hace hincapié en escuelas innovadoras en el sistema educativo ecuatoriano. Los resultados de las CPA fueron altos en 3,91, y la eficacia pedagógica fue comparativamente alta en 3,54. En general, las escuelas de Azogues y Cuenca hacen hincapié en las escuelas de innovación, lo que indica que hay cambios en este campo.

\section{Análisis de las diferencias en fun- ción de las características de los profesores}

\section{Cultura organizativa escolar}

Los resultados de examinar si hay una diferencia en la COE, según los antecedentes personales del profesor se presentan en la tabla 2 .

La COE percibida por los profesores mostró una diferencia significativa en la de la innovación, la racional, la de grupo y la de la jerarquía, según las notas de la institución en un promedio de la escala de cinco puntos. En el nivel escolar, los maestros de la escuela primaria percibían la cultura de la innovación más alto que sus pares de la escuela básica y general unificado, mientras que las racionales y jerárquicas eran más bajas.

Los profesores de básica percibían culturas racionales y jerárquicas más altas que los docentes de inicial y general unificado. En cuanto al género, los masculinos la observaban más que las femeninas, mientras que ellas percibían la cultura de la innovación

Tabla 2. Análisis de la cultura organizativa escolar, según los antecedentes personales del profesor

\begin{tabular}{|c|c|c|c|c|c|c|c|c|c|c|c|c|c|c|}
\hline \multirow[b]{2}{*}{ Variable } & \multirow{2}{*}{$\begin{array}{r}\begin{array}{r}\text { Cultura } \\
\text { organizacional }\end{array} \\
\end{array}$} & \multirow{2}{*}{$\mathbf{N}$} & \multicolumn{3}{|c|}{$\begin{array}{l}\text { Cultura de la } \\
\text { innovación }\end{array}$} & \multicolumn{3}{|c|}{$\begin{array}{l}\text { Cultura } \\
\text { racional }\end{array}$} & \multicolumn{3}{|c|}{ Cultura grupal } & \multicolumn{3}{|c|}{$\begin{array}{l}\text { Cultura de } \\
\text { jerarquía }\end{array}$} \\
\hline & & & $\sigma$ & $\sigma$ & $\mathbf{F}$ & $б$ & $\sigma$ & $\mathbf{F}$ & 6 & $\boldsymbol{\sigma}$ & $\mathbf{F}$ & 6 & $\sigma$ & $\mathbf{F}$ \\
\hline \multirow{4}{*}{ Nivel escolar } & Inicial & 3 & 4.2 & .61 & \multirow{4}{*}{2.1} & 2.2 & .81 & \multirow{4}{*}{18.1} & 4.38 & .52 & & 1.6 & .52 & \multirow{4}{*}{41.8} \\
\hline & Básica & 71 & 3.8 & .60 & & 2.5 & .77 & & 4.00 & .61 & 270 & 2.3 & .86 & \\
\hline & General unificado & 244 & 3.5 & .69 & & 2.9 & .76 & & 3.65 & .68 & 21.0 & 2.8 & .79 & \\
\hline & Total & 358 & 3.6 & .70 & & 2.7 & .81 & & 3.81 & .69 & & 2.5 & .86 & \\
\hline \multirow{2}{*}{ Género } & M & 162 & 3.5 & .64 & \multirow{2}{*}{-2.5} & 2.9 & .72 & \multirow{2}{*}{2.7} & 3.76 & .65 & 10 & 2.7 & .79 & \multirow{2}{*}{3.8} \\
\hline & $\mathrm{F}$ & 195 & 3.7 & .74 & & 2.6 & .86 & & 3.88 & .72 & -1.9 & 2.4 & .89 & \\
\hline \multirow[b]{2}{*}{ Nivel } & Profesores de escuela & 253 & 3.6 & .70 & \multirow[b]{2}{*}{-2.8} & 2.7 & .80 & \multirow[b]{2}{*}{-1.0} & 2.79 & .80 & & 2.6 & .86 & \multirow[b]{2}{*}{2.5} \\
\hline & $\begin{array}{l}\text { Director (rector de } \\
\text { escuela) }\end{array}$ & 103 & 3.8 & .66 & & 2.6 & .82 & & 2.69 & .82 & -1.8 & 2.4 & .86 & \\
\hline \multirow{5}{*}{$\begin{array}{l}\text { Experiencia } \\
\text { docente }\end{array}$} & Menos de 5 años & 90 & 3.5 & .77 & \multirow{5}{*}{4.4} & 2.9 & .97 & \multirow{5}{*}{3.8} & 3.77 & .70 & & 2.8 & .82 & \multirow{5}{*}{6.6} \\
\hline & 6-15 años & 120 & 3.6 & .70 & & 2.7 & .72 & & 3.75 & .71 & & 2.5 & .84 & \\
\hline & 16-25 años & 72 & 3.9 & .66 & & 2.5 & .83 & & 3.98 & .75 & 2.0 & 2.2 & .89 & \\
\hline & Más de 26 años & 75 & 3.7 & .60 & & 2.8 & .64 & & 3.77 & .56 & & 2.6 & .84 & \\
\hline & Total & 357 & 3.6 & 70 & & 2.7 & .81 & & 3.81 & .69 & & 2.58 & .86 & \\
\hline
\end{tabular}


más que ellos. Según el cargo, los directores de escuela percibían la cultura de la innovación más alto que los profesores titulares. Además, según los años de experiencia docente, quienes tenían menos de 5 años de trayectoria poseían una gran conciencia de las racionales y jerárquicas, mientras que aquellos con 16 a 25 años de práctica reconocían la de innovación y de grupo.

\section{Comunidad profesional de aprendizaje}

Los resultados de examinar si hay una diferencia en la CPA según los antecedentes personales del profesor se muestra en la siguiente tabla 3 .

En los resultados se visualiza la escala de 5 puntos, la CPA de la organización escolar fue de 3,91. Hubo una diferencia significativa en los grados escolares, lo que indica que los maestros de la escuela de nivel inicial perciben las CPA más alto que los maestros de la básica y general unificado o bachillerato.
También, hubo diferencias significativas en cuanto al género, ya que las profesoras percibían niveles más altos de CPA que los profesores. Además, los docentes que participaban en la CPA percibían la comunidad de aprendizaje más que los que no participaban.

\section{Eficacia pedagógica de los docentes}

Las diferencias en la eficacia pedagógica de los profesores según sus antecedentes se presentan en la tabla 4.

En promedio, la eficacia pedagógica de los profesores fue de 3,54 en la escala de 5 puntos. Al revisar sus características personales, hubo una diferencia significativa en las calificaciones escolares. Los docentes de la escuela básica percibían una mayor eficacia pedagógica del profesorado que los de la escuela de nivel general unificado.

Además, hubo una diferencia significativa en el nivel de los profesores; el director (rector) percibió la eficacia pedagógica de los profesores más alta que la

Tabla 3. Análisis de la diferencia en la comunidad profesional de aprendizaje según los antecedentes personales del profesor

\begin{tabular}{llccccc}
\hline Categoría & \multicolumn{1}{c}{ Estadísticas } & N & $\sigma$ & $\boldsymbol{\sigma}$ & F & $\begin{array}{c}\text { Prueba de } \\
\text { Scheffé }\end{array}$ \\
\hline Nivel escolar & Inicial & 42 & 4.55 & .42 & & E $>$ M, H \\
& Básica & 71 & 4.10 & .47 & 37.97 & $\mathrm{M}>\mathrm{H}$ \\
& General unificado & 238 & 3.74 & .64 & & \\
Género & Total & 351 & 3.91 & .64 & & \\
& $\mathrm{M}$ & 159 & 3.75 & .57 & -4.29 & \\
Nivel & F & 191 & 4.04 & .68 & & \\
Experiencia & Profesores de escuela & 249 & 3.88 & .65 & -1.47 & \\
docente & Director (rector de escuela) & 100 & 3.99 & .63 & & \\
& Menos de 5 años & 89 & 3.78 & .64 & & \\
& De 6-15 años & 117 & 3.93 & .66 & & \\
& De 16-25 años & 70 & 4.09 & .70 & 3.24 & $16-25>5$ \\
& 26 años o más & 74 & 3.85 & .52 & & \\
\hline
\end{tabular}


Tabla 4. Análisis de las diferencias en la eficacia pedagógica de los profesores según sus antecedentes personales

\begin{tabular}{|c|c|c|c|c|c|c|}
\hline Categoría & Estadística & $\mathbf{N}$ & 6 & $\boldsymbol{\sigma}$ & $\mathbf{F}$ & $\begin{array}{c}\text { Prueba de } \\
\text { Scheffe } \\
\end{array}$ \\
\hline \multirow[t]{4}{*}{ Nivel escolar } & Inicial & 40 & 3.75 & .53 & \multirow{4}{*}{3.47} & \multirow{6}{*}{$\mathrm{E}>\mathrm{H}$} \\
\hline & Básica & 71 & 3.55 & .48 & & \\
\hline & General unificado & 237 & 3.50 & .56 & & \\
\hline & Total & 348 & 3.54 & .55 & & \\
\hline \multirow[t]{2}{*}{ Género } & Masculino & 156 & 3.57 & .54 & \multirow{2}{*}{1.15} & \\
\hline & Femenino & 191 & 3.51 & .56 & & \\
\hline \multirow[t]{2}{*}{ Nivel } & Profesores de escuela & 246 & 3.47 & .56 & \multirow{2}{*}{-3.47} & \\
\hline & Director (rector de escuela) & 100 & 3.70 & .49 & & \\
\hline \multirow{5}{*}{$\begin{array}{l}\text { Experiencia } \\
\text { docente }\end{array}$} & Menos de 5 años & 89 & 3.34 & .57 & \multirow{5}{*}{9.14} & \multirow{5}{*}{$\begin{array}{l}16-25>5 \\
6-15 \text { años }\end{array}$} \\
\hline & De 6-15 años & 114 & 3.48 & .56 & & \\
\hline & De $16-25$ años & 71 & 3.73 & .51 & & \\
\hline & 26 años o más & 73 & 3.67 & .46 & & \\
\hline & Total & 347 & 3.54 & .55 & & \\
\hline
\end{tabular}

de los profesores normales. Asimismo, los maestros de 16 a 25 años percibían la eficacia pedagógica de los docentes más alta que los maestros con 6 a 15 años de experiencia, como también los que tienen menos de 5 años (tabla 4).

\section{Relación entre las tres variables de la investigación}

\section{El efecto de la cultura organizativa de la escuela en la eficacia de los maestros}

Los resultados del análisis del efecto de la COE en la eficacia de los maestros son los siguientes:
La cultura de la innovación y la de grupo de la organización escolar tuvieron un impacto significativo en la eficacia de los maestros en un $1 \%$. Se encontró que el tipo de COE explicaba alrededor del $21 \%$ de la varianza de la eficacia de los docentes, considerándose que el peso relativo de la eficacia pedagógica de los docentes entre los tipos de cultura organizativa era el coeficiente $\beta$ (coeficiente de regresión estandarizada), que mostraba que la cultura de grupo seguía la cultura de la innovación (tabla 5).

Tabla 5. El efecto de la cultura organizativa escolar en la eficacia.

\begin{tabular}{|c|c|c|c|c|c|c|}
\hline $\begin{array}{c}\text { Variable } \\
\text { dependiente }\end{array}$ & Variable independiente & $\begin{array}{l}\text { Coeficiente no } \\
\text { estandarizado }\end{array}$ & $\begin{array}{c}\text { Coeficiente } \\
\text { estandarizado }(\beta) \\
\end{array}$ & $\mathbf{T}$ & $\mathbf{F}$ & $\mathbf{R}^{2}$ \\
\hline \multirow[t]{5}{*}{ Eficacia pedagógica } & (constante) & 1.61 & & 6.41 & & \\
\hline & Cultura de la innovación & .278 & .353 & 5.44 & & \\
\hline & Cultura racional & .040 & .059 & 1.07 & 22.49 & .211 \\
\hline & Cultura de grupo & .160 & .203 & 3.20 & & \\
\hline & Cultura de jerarquía & .070 & .110 & 1.70 & & \\
\hline
\end{tabular}

$* \mathrm{p}<.05 * * \mathrm{p}<.01$ 


\section{El efecto de las comunidades profesionales de aprendizaje en la eficacia pedagógica de los docentes}

Los efectos de las comunidades profesionales de aprendizaje en la eficacia pedagógica de los docentes se muestran en la tabla 6 .

Entre los subfactores de la CPA, se encontró que el que tiene un efecto significativo en la eficacia, es la promoción del aprendizaje y la práctica con un $1 \%$, que es un predictor significativo de la eficacia pedagógica de los maestros. El factor más bajo de la CPA representa alrededor del $22 \%$ de la varianza de la eficacia pedagógica de los maestros, y el peso relativo de la eficacia de los maestros entre los factores más bajos de la CPA fue el coeficiente $\beta$ (coeficiente de regresión estandarizada), que fue la práctica, seguido de la promoción del aprendizaje (tabla 6).

\section{El efecto de la cultura organizativa de las escuelas y las comunidades profesionales de aprendizaje en la eficacia de los docentes}

Los resultados del análisis de los efectos generales de las subvariables de la $\mathrm{COE}$ y la CPA sobre la eficacia pedagógica de los docentes se muestran en la tabla 7.

Entre las subculturas, la COE, la cultura de la innovación y las subvariantes de la CPA tienen un efecto significativo en la eficacia pedagógica de los profesores. Por

Tabla 6. El efecto de las comunidades profesionales de aprendizaje en la eficacia de

\begin{tabular}{|c|c|c|c|c|c|c|}
\hline $\begin{array}{c}\text { Variable } \\
\text { dependiente }\end{array}$ & Variable independiente & $\begin{array}{l}\text { Coeficiente no } \\
\text { estandarizado }\end{array}$ & $\begin{array}{c}\text { Coeficiente } \\
\text { estandarizado }(\beta)\end{array}$ & $\mathbf{T}$ & $\mathbf{F}$ & $\mathbf{R}^{2}$ \\
\hline \multirow{5}{*}{$\begin{array}{l}\text { Eficacia pedagógica } \\
\text { del docente }\end{array}$} & (constante) & 1.981 & & 10.48 & \multirow{5}{*}{24.25} & \multirow{5}{*}{.224} \\
\hline & Promoción del aprendizaje & .070 & .309 & 3.64 & & \\
\hline & Cultura cooperativa & -.034 & -.158 & -1.62 & & \\
\hline & Grupo de investigación & -.004 & -.037 & -0.34 & & \\
\hline & Práctica pedagógica & .028 & .360 & 3.21 & & \\
\hline
\end{tabular}

Tabla 7. El efecto de la cultura organizacional escolar y las comunidades profesionales de aprendizaje en la eficacia pedagógica de los docentes

\begin{tabular}{|c|c|c|c|c|c|c|c|}
\hline $\begin{array}{c}\text { Variable } \\
\text { dependiente }\end{array}$ & Varia & independiente & $\begin{array}{l}\text { Coeficiente no } \\
\text { estandarizado }\end{array}$ & $\begin{array}{c}\text { Coeficiente } \\
\text { estandarizado }(\beta)\end{array}$ & $\mathbf{T}$ & $\mathbf{F}$ & $\mathbf{R}^{2}$ \\
\hline \multirow{9}{*}{$\begin{array}{l}\text { Capacidad } \\
\text { profesional } \\
\text { docente }\end{array}$} & \multirow{5}{*}{$\begin{array}{l}\text { Organización } \\
\text { cultural escolar }\end{array}$} & (constante) & 1.293 & & 4.69 & \multirow{9}{*}{15.02} & \multirow{9}{*}{.269} \\
\hline & & Cultura de la innovación & .177 & .225 & 3.21 & & \\
\hline & & Cultura racional & .039 & .058 & 1.07 & & \\
\hline & & Cultura organizacional & .077 & .098 & 1.38 & & \\
\hline & & Cultura de la jerarquía & .063 & .098 & 1.56 & & \\
\hline & \multirow{4}{*}{$\begin{array}{l}\text { Comunidad } \\
\text { profesional de } \\
\text { aprendizaje }\end{array}$} & $\begin{array}{l}\text { Promoción del } \\
\text { aprendizaje }\end{array}$ & .059 & .257 & 3.02 & & \\
\hline & & Cultura cooperativa & -.030 & -.141 & -1.43 & & \\
\hline & & Grupo de investigación & -.011 & -.092 & -.84 & & \\
\hline & & Práctica pedagógica & .022 & .278 & 2.45 & & \\
\hline
\end{tabular}


otro lado, en general, la COE y la CPA explica alrededor del $27 \%$ de la varianza de la eficacia de los profesores. Como resultado de la comparación de las influencias relativas de las variables sobre la eficacia, se comprobó que tenían una influencia significativa en el orden de la práctica pedagógica, la promoción del aprendizaje y la cultura de la innovación (tabla 7).

\section{Efecto de la cultura organizativa de la escuela y la comunidad profe- sional de aprendizaje en la eficacia}

Los resultados sobre la eficacia pedagógica de la COE y CPA de los docentes en diferentes etapas, se muestran en la tabla 8 . Se pudo observar que la CPA cuenta con un valor mayor de .269 , tanto en eficacia pedagógica docente como de potencia explicativa, al contrario de la $\mathrm{COE}$ con un valor menor de .211. Por lo tanto, la CPA puede considerarse un menor predictor de la eficacia pedagógica del docente que una COE.

\section{CONCLUSIONES}

El propósito de este estudio fue analizar la eficacia pedagógica entre la COE y la CPA. Los resultados del estudio mostraron que había una diferencia significativa en la COE por subtipo en el nivel, el género, el profesorado y la experiencia; también, lo hubo en las CPA, en los mismos factores $\mathrm{y}$, en cuanto a la eficacia pedagógica de los profesores, hubo una diferencia significativa en cuanto al nivel escolar, nivel de los profesores y experiencia docente.

El examen de la influencia de la $\mathrm{COE}$ y las CPA en la eficacia pedagógica de los docentes, demostró que la cultura de la innovación y la de organización o de grupo influían en la escolaridad, y que estas comunidades afectaban a la formación del aprendizaje y la práctica pedagógica. También se comprobó que la cultura de las escuelas tenía una mayor influencia en la eficacia pedagógica que dichas comunidades.

La escuela es un tipo de sistema organizativo, pero los docentes demostraron aspectos diversos y variados. Los profesores de género masculino tienden a ver la organización escolar como algo más burocrático al contrario de las profesoras. Seguidamente, las profesoras percibieron la organización escolar, como una cultura de innovación, además, perciben más la CPA que los profesores.

En cuanto a la eficacia pedagógica, había diferencias en el nivel escolar y el del profesorado, pero no por género. Los docentes de las escuelas de básica tendían a tener una mayor eficacia pedagógica y los directores a ser más eficaces que los maestros ordinarios. En consecuencia, los maestros responden a la organización escolar de varias maneras, según sus características personales.

De igual forma, la COE se está convirtiendo gradualmente, en una estructura

Tabla 8. Etapas del efecto de la cultura organizacional escolar y de la comunidad profesional de aprendizaje sobre la eficacia de los maestros

\begin{tabular}{lcccc}
\hline \multicolumn{1}{c}{ Categoría } & $\mathbf{R}^{2}$ & $\mathbf{F}$ & $\mathbf{R 2}$ & F \\
\hline Escenario 1. & .211 & 22.49 & & \\
Variable: cultura organizacional escolar & & & & \\
Escenario 2. & .269 & 15.02 & .058 & 10.52 \\
Variable: comunidad profesional de aprendizaje & &
\end{tabular}


saludable. Entre sus tipos, la de la innovación y la de grupo u organizacional, tuvieron un efecto significativo en la eficacia pedagógica de los profesores. Sin embargo, la de la innovación y la de grupo fueron superiores a las racionales y a las de jerarquía.

Las culturas de la innovación y de grupo tienen un efecto positivo en la eficacia de los profesores, porque hacen hincapié en la coordinación, la cohesión, el trabajo en equipo y la colaboración entre ellos. Este resultado, se debe al cambio de las culturas de los docentes en el último periodo académico, que hace hincapié en las escuelas de innovación ecuatorianas.

La CPA de los docentes tiene un efecto positivo en la eficacia pedagógica. Se está convirtiendo en un factor importante para mejorar la enseñanza, al promover el crecimiento y el aprendizaje de los estudiantes, como también, al realizar mejoras continuas mientras hacen las prácticas pedagógicas. Puede decirse que esto representa el hecho, de que se han adoptado diversas medidas para aumentar el rendimiento de los alumnos a medida que los profesores acrecientan su profesionalismo y experiencia.

La COE y la CPA deben ser discutidas más allá del nivel de interacción e integración, para mejorar la eficacia pedagógica de los docentes y como elementos importantes de la mejora de la escuela. Esta cultura está incrustada en creencias y valores, normas y costumbres, hábitos y rituales que son compartidos universalmente, por los integrantes y sirve de guía para el comportamiento de quienes la componen.

Por lo tanto, la COE juega un papel importante en el refuerzo de la voluntad de los profesores de seguir las instrucciones del director (rector). Especialmente, cuando se ejerce una cultura de innovación y de grupo, la CPA se vuelve más activa cuando estos elementos se combinan, luego la eficacia pedagógica del docente aumenta. En este contexto, es necesario crear una cultura escolar y hacer hincapié en la CPA en el nivel organizativo como factor de cambio. De hecho, vale recordar que la eficacia pedagógica de los docentes, aumenta cuando las CPA trabajan juntas en lugar de la COE por sí sola.

\section{DECLARACIÓN DE LA CONTRIBUCIÓN DE LOS AUTORES}

El porcentaje total de contribución para la conceptualización, la preparación y la corrección de este artículo fue el siguiente: D. J. M. V. $50 \%$, M. F. C. M. 12,5\%, M. N. C. $12,5 \%$, E. M. F. H. $12,5 \%$ y K. M. C. P. $12,5 \%$.

\section{DECLARACIÓN DE DISPO- NIBILIDAD DE LOS DATOS}

Los datos que respaldan los resultados de este estudio serán puestos a disposición por el autor correspondiente D. J. M. V., previa solicitud razonable.

\section{REFERENCIAS}

Aditomo, A., \& Klieme, E. (2020). Forms of inquiry-based science instruction and their relations with learning outcomes: evidence from high and low-performing education systems. International Journal of Science Education, 42(4), 504-525. doi: https://doi.org/10.1080/ 09500693.2020.1716093

Areepattamannil, S. (2012). Effects of inquiry-based science instruction on science achievement and interest in science: Evidence from Qatar. The Journal of Educational Research, 105(2), 134-146. doi: https://doi.org/10.1080/00220 671.2010 .533717 
Aydeniz, M., \& Dogan, A. (2016). Exploring pre-service science teachers' pedagogical capacity for formative assessment through analyses of student answers. Research in Science \& Technological Education, 34(2), 125-141. https:// doi.org/10.1080/02635143.2015.1092954

Benedict, A., \& Aram, R. (2017). Tools for teacher noticing: Helping preservice teachers notice and analyze student thinking and scientific practice. Journal of Science Teacher Education, 28(3), 294-318. https://doi. org/10.1080/1046560X.2017.1302730

DuFour, R.; Eaker, R., \& Many, T. (2010). Learning by doing. A handbook for professional learning communities at work. Bloomington: Solution Tree Press.

Elkader, N. (2014). Enfoques epistemológicos de la enseñanza dialógica en un entorno convencional: revisión crítica. Dialogic Pedagogy Journal, 2, 1-7. doi: https://doi.org/10.5195/dpj.2014.83

Fogleman, J.; McNeill, K. L., \& Krajcik, J. (2011). Examining the effect of teachers' adaptations of a middle school science inquiry-oriented curriculum unit on student learning. Journal of Research in Science Teaching, 48(2), 149 169. doi: https://doi.org/10.1002/tea.20399

Huopalainen, A. (2019). Manipulating surface and producing 'effortless' elegance - analysing the social organization of glamour. Culture and Organization, 25(5), 332-352. doi: https://doi. org/10.1080/14759551.2016.1264949

Kivunja, Ch., \& Bawa Kuyini, K. (2017). Understanding and Applying Research Paradigms in Educational Contexts. International Journal of Higher Education, 6(5), 26-41. doi: https:// doi.org/10.5430/ijhe.v6n5p26

Lee, Y. (1997). Relationship between school organizational culture, principal's instructional leadership, and teacher's professional capacity. Daejeon: Chungnam National University.

Lee, S. Y. (2007). Teachers' Team Learning Activity Analysis and Learning Organization Relationships. Korean Journal of Educational Administration, 25(4), 95-115.
Mendoza, D.; Cejas, M.; Navarro, M.; Vega, V., \& Albán, C. (2019a). Software de investigación Moodle: contexto emocional en la educación superior ecuatoriana. International Journal of Engineering Research and Technology, 12(9), 1491-1500.

Mendoza, D.; Abrigo, I.; Romero, J.; Cueva, F., \& Cejas, M. (2019b). The formative research of ecuadorian university teaching staff. Problems of education in the 21stcentury, 77(3), 364-378. doi: https://doi.org/10.33225/pec/19.77.364

Robin, A. (2004). Hacia la enseñanza dialógica: repensar la charla en el aula. Cambridge: Diálogos.

Rodríguez, S.; Núñez, J.; Valle, A.; Blas, R., \& Rosario, P. (2009). Autoeficacia docente, motivación del profesor y estrategias de enseñanza. Escritos de Psicología, 3(1), 1-7.

Ross, J., \& Bruce, C. (2007). Professional development effects on teacher efficacy: Results of randomized field trial. The journal of educational research, 101(1), 50-60. https://doi. org/10.3200/JOER.101.1.50-60

Senge, P. M., Cambron-McCabe, N., Lucas, T., Smith, B., Dutton, J., \& Kleiner, A. (2000). Schools that learn. New York: Doubleday.

Vinhote, J. Gomes, J. Rodrigues, N. Sena, E. \& de Sousa, D. (2020). Diagnóstico de accesibilidad en las aulas FT / UFAM. Revista Internacional de Innovación, Educación e Investigación, 8(3), 215-234. doi: https://doi.org/10.31686/ijier.vol8.iss3.2224

Wieslander, M. (2019). Challenging and destabilizing official discourses: irony as a resistance resource in institutional talk. Culture and $\mathrm{Or}$ ganization, 26(3), 13-34. doi: https://doi.org/ 10.1080/14759551.2019.1699093

Zaigraev, A., \& Wilk, M. (2020). Optimal designs for heteroscedastic regression models with two parameters. Statistics, 54(2), 291-309. doi: https:// doi.org/10.1080/02331888.2019.1702041

Zanin, A., \& Bisel, R. (2020). Concertive resistance: how overlapping team identifications enable collective organizational resistance. Culture and Organization, 26(3), 231-249. doi: https:// doi.org/10.1080/14759551.2019.1566233

\section{(c) $(1) \Theta$}

La eficacia pedagógica en la cultura organizativa escolar y la comunidad profesional de aprendizaje (Derling José Mendoza Velazco • Magda Francisca Cejas Martínez • Mercedes Navarro Cejas • Elizeth Mayrene Flores Hinostroza $•$ Karina Maribel Castillo Pinos) Uniciencia is protected by Attribution-NonCommercial-NoDerivs 3.0 Unported (CC BY-NC-ND 3.0) 